\title{
Mixed convection boundary-layer stagnation point flow past a vertical stretching/shrinking surface in a nanofluid
}

\begin{abstract}
A steady two dimensional mixed convection boundary layer flow near a stagnation point on a impermeable vertical stretching/shrinking surface in a nanofluid with controlled nanoparticles volume fraction is investigated numerically. The stretching/shrinking velocity and the ambient fluid velocity are assumed to vary linearly with the distance from the stagnation-point. The governing partial differential equations are first transformed into ordinary differential equations using a similarity transformation, before being solved numerically using a shooting method. The effects of several physical parameters such as mixed convection parameter, stretching/shrinking parameter, Brownian motion parameter, thermophoresis parameter and Lewis number on the skin friction coefficient, heat and mass transfer rates at the surface as well as the velocity, temperature and concentration profiles are analysed and discussed. It was found that increasing the particular values of the mixed convection parameter tends to increase the skin friction coefficient, heat and mass transfer rates at the surface. The results obtained show that the solution is unique for the stretching surface whereas dual (upper and lower branch) solutions exist for the shrinking surface. It should be mentioned that the solutions obtained are only locally similar.
\end{abstract}

Keyword: Mixed convection; Nanofluid; Stretching/shrinking; Stagnation point 